\title{
Case Report \\ Continuous Spinal Anaesthesia for Intertrochanteric Femur Fracture in a Patient with Skeletal Dysplasia
}

\author{
Sharad Khakurel (iD and Rupesh Kumar Yadav \\ National Academy of Medical Sciences, National Trauma Centre, Kathmandu, Nepal \\ Correspondence should be addressed to Sharad Khakurel; sharadsarad@hotmail.com
}

Received 21 December 2020; Revised 8 March 2021; Accepted 3 April 2021; Published 13 April 2021

Academic Editor: Serkan Tulgar

Copyright (c) 2021 Sharad Khakurel and Rupesh Kumar Yadav. This is an open access article distributed under the Creative Commons Attribution License, which permits unrestricted use, distribution, and reproduction in any medium, provided the original work is properly cited.

\begin{abstract}
The practice of continuous spinal anaesthesia is not common. Though underutilised, it offers significant advantage when compared to the single-shot technique nonetheless. Time and again, it has proven its worth in patients with advanced cardiac illness, spinal deformities, and obesity. We here successfully employed this neuraxial anaesthetic technique in a sixty-two-year-old male patient with skeletal dysplasia, who presented for surgical fixation of intertrochanteric fracture of the femur. With short stature, anticipated difficult airway, and poor pulmonary status complicating the anaesthetic plan, we opted for continuous spinal anaesthesia. The procedure was carried out uneventfully with $8 \mathrm{mg}$ of hyperbaric bupivacaine used in titration to anaesthetic needs. Patients with skeletal dysplasia present with wide array of clinical conditions that pose a formidable challenge to anaesthesiologists. Continuous spinal anaesthesia can be safely practiced in such patients as it provides a titratable form of neuraxial blockade with reduced dose of local anaesthesia. This, in turn, ensures a predictable block and, thus, hemodynamic stability.
\end{abstract}

\section{Introduction}

Skeletal dysplasia encompass a heterogeneous group of disorders that affect the development of bones and cartilage. Its estimated incidence is approximately 15.7 in 100,000 births and most occur as a result of genetic defects. It commonly presents during childhood and is suspected when a patient has short stature, bony deformities, and recurrent fractures [1]. Consequently, the aberrant growth of the long bones, spine, and skull bones leads to morphological abnormalities in the body. Although a disorder of musculoskeletal system, they can also be complicated by involvement of cardiorespiratory and neurologic systems [2].

Patients with skeletal dysplasia may often present with fractures requiring surgical correction. Anaesthetic management of such patients is not without challenges. Deformed skull and facial bones along with vertebral anomalies present with an exacting airway scenario [3-7]. Spinal stenosis and cervical spine instability are common in these patients and warrants extreme caution, especially during laryngoscopy in order to prevent cervical cord compression [3-7]. Thoracic kyphoscoliosis, chest wall deformities with pulmonary involvement, and recurrent respiratory infections are common [7] which makes general anaesthesia a less-preferred option. The spine is also affected by various degrees of kyphoscoliosis and lumbar lordosis; thus, performing neuraxial anaesthesia may become technically difficult $[3,8]$. We discuss here an anaesthetic management of one such patient planned for proximal femoral nailing by the use of Continuous Spinal Anaesthesia (CSA).

\section{Case Presentation}

A 62-year-old male was referred to our centre after sustaining injury to his left leg in a landslide. On arrival in the emergency department (ED), his Glasgow Coma Scale (GCS) was 15 with a pulse rate of 88 beats per minute and blood pressure of 120/80 millimetre $(\mathrm{mm})$ of mercury $(\mathrm{Hg})$. The oxygen saturation in room air was $85 \%$ with respiratory rate of 20 per minute. He was subjected to trauma imaging, 
and an X-ray of his left pelvis revealed intertrochanteric fracture of femur (Figure 1). A workup for the patients low oxygen saturation was performed, as there was no history of acute or chronic respiratory illness. On auscultation, there were diminished breath sounds on the right chest. Chest radiography was performed which showed heterogeneous infiltrations in his right lung, with crowding of the ribs and scoliotic spine with narrow intervertebral space (Figure 2). A nasopharyngeal swab for Reverse Transcriptase Polymerase Chain Reaction (RT PCR) for Severe Acute Respiratory Syndrome Coronavirus 2 (SARS-CoV-2) was collected. He was managed in the ED with oxygen via a nasal prong at 3 litres per minute, antibiotics, analgesics, and incentive spirometry and skin traction on the affected limb. The patient was planned for operative intervention and stabilisation of the fracture by proximal femoral nailing.

During the preoperative assessment of the patient, it was revealed that the patient was a known case of skeletal dysplasia. No further inquiry to ascertain the type of dysplasia was conducted previously. Both his son and daughter also suffered from skeletal dysplasia. The patient was a farmer by profession and had good functional status with a metabolic equivalent of more than four. He had a short stature with a body length of 127 centimetres $(\mathrm{cm})$ (Figure 3) and weight of 45 kilograms $(\mathrm{kg})$. On airway evaluation, the patient had adequate mouth opening with an interincisor distance of $4 \mathrm{~cm}$, Mallampati class III, and missing multiple teeth. He had a short neck with a sternomental distance of $10 \mathrm{~cm}$ and thryomental distance of $4.5 \mathrm{~cm}$. On systemic examination, a chest deformity, pectus carinatum, (Figure 4) was visible with a small rib cage. His spine was also examined and had dorsolumbar scoliosis with narrow intervertebral space. Rest systemic examination findings were within normal limits. The lab reports were also within normal limits. A pulmonary function test (PFT) was sought with restrictive lung disease in mind, and an echocardiography was ordered to assess the effect of restrictive lung disease on the heart. Echocardiography showed mild tricuspid regurgitation and mild pulmonary arterial hypertension with a pulmonary artery systolic pressure of $44 \mathrm{~mm}$ of $\mathrm{Hg}$ with an ejection fraction of $60 \%$. Due to technical issues, the PFT could not be performed.

The patient was planned for regional anaesthesia considering the difficult airway and pulmonary issues. CSA was opted following counselling of the patient and after acquisition of informed written consent. On the day of surgery, the patient was shifted to the operating theatre, and standard American Society of Anaesthesiologists (ASA) monitoring was performed. Injection (Inj.) cefuroxime 750 grams was infused as a part of the infection prevention protocol. A paediatric Tuohy epidural needle 19 gauge was used in an attempt to access subarachnoid space. The procedure, however, was technically very challenging due to positional pain and spine anatomy. During the needling, a whitish discharge was noted from the skin puncture site, which was sent for microbiological examination, and the procedure was abandoned. A spine MRI was performed for further evaluation. It, however, did not reveal any infective pathology, but there was diffuse disc bulge along with disc desiccation of

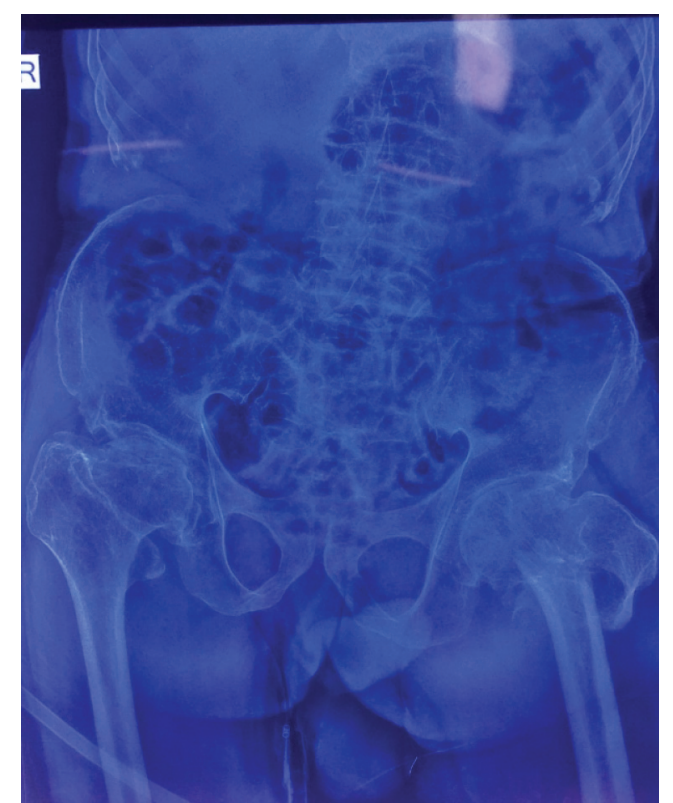

FIgURE 1: Intertrochanteric fracture of the femur (left).

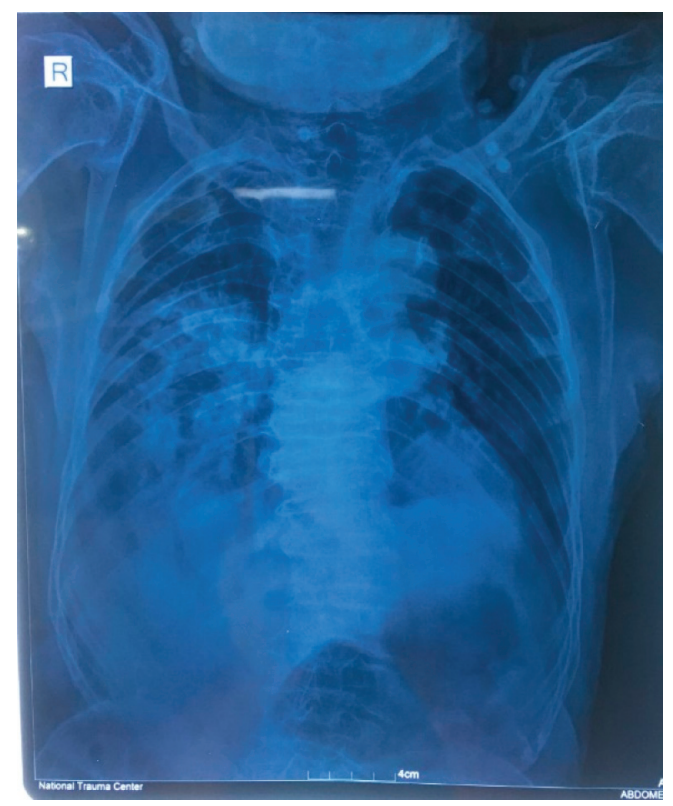

Figure 2: Chest X-ray.

all visible intervertebral discs. A dorsolumbar scoliosis with cervico-dorsal-lumbar spondylosis was also noted.

After observation for 48 hours, the patient was scheduled once again for the contemplated procedure, as microbiological examination was negative for any infective pathology.

In the theatre, the patient was the given Inj. midazolam $1 \mathrm{mg}$ and Inj. fentanyl $50 \mathrm{mcg}$. Ultrasound guided lumbar plexus block and trans-gluteal sciatic nerve block were performed in the lateral position to provide analgesia for positioning of the patient for CSA and also as a backup in case the planned spinal anaesthesia failed. After twenty minutes of the block, the patient was positioned for spinal anaesthesia in the sitting position. This time, the numeric 


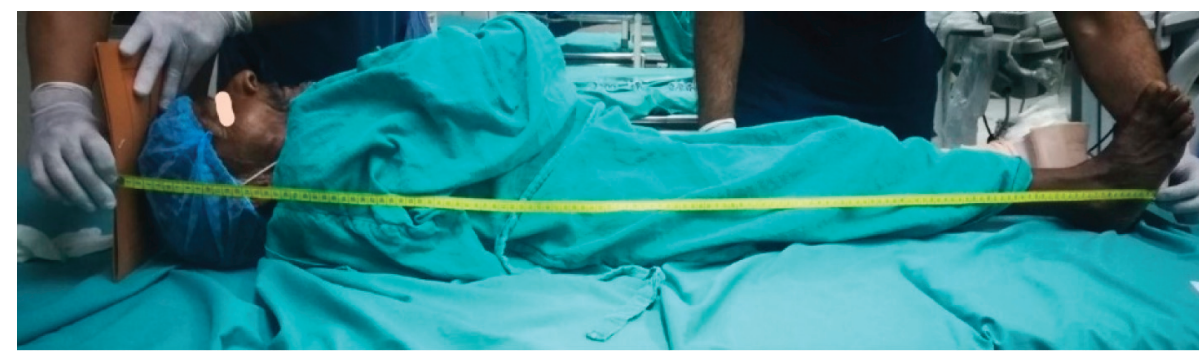

Figure 3: Short stature of the patient.

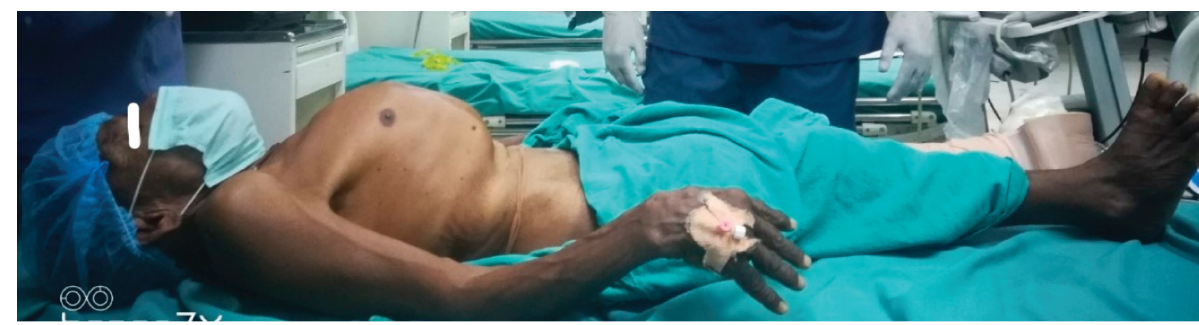

FIGURE 4: Obvious chest deformity.

rating scale for positional pain was 3 out of 10 . With the help of a paediatric Tuohy needle, the dura was punctured at $3 \mathrm{~cm}$ depth and free flow of the cerebrospinal fluid (CSF) was observed. Then, a paediatric epidural catheter 22 gauge was inserted and fixed at $6 \mathrm{~cm}$. Backflow of CSF was checked, and 0.4 millilitres ( $\mathrm{ml}$ ) ( $2 \mathrm{mg}$ ) of hyperbaric $0.5 \%$ Bupivacaine was administered over a minute. The effect of spinal anaesthesia was confirmed on the patient's normal leg. Approximately after eight minutes, the level of sensory block reached T8. Surgery was then allowed to commence as the motor blockade measured by the modified Bromage score reached 2. During the two hours of surgical duration, a single episode of hypotension was noted which resolved with fluid bolus. Based on the assessment of block height regression, the patient received further three aliquots of $0.4 \mathrm{ml}$ of hyperbaric bupivacaine. So, the entire procedure was completed with $8 \mathrm{mg}$ of $0.5 \%$ hyperbaric bupivacaine. We removed the catheter in the postoperative unit after infusing another $0.4 \mathrm{ml}$ of hyperbaric bupivacaine. The catheter was removed as the expertise to handle CSA in the postoperative ward was inadequate. The patient was discharged from postoperative care after 24 hours of uneventful stay.

\section{Discussion}

Hip fractures are commonly managed surgically under neuraxial or general anaesthesia (GA). While both techniques have their pros and cons, convincing evidence of one's superiority over the other is lacking. A Cochrane review pointed out the only benefit of neuraxial anaesthesia over GA lies in lowering the risk of deep vein thrombosis, when anticoagulant prophylaxis is not used [9]. The International Consensus on Anaesthesia-Related Outcomes after Surgery review on anaesthesia for elective hip arthroplasty recommended neuraxial anaesthesia in terms of better perioperative outcomes [10].
Single-shot spinal anaesthesia (SSA) and continuous epidural anaesthesia (CEA) are frequently used neuraxial approaches for management of hip fractures. Continuous spinal anaesthesia (CSA), though underutilised, is a wellestablished neuraxial technique. It safely offers reliable and dense anaesthesia with small doses of local anaesthetic that can be easily titrated. As a result, the block is well predicted and tends to cause less hemodynamic perturbations [11, 12]. Hence, it forms the basis for usage of CSA in high-risk cases $[11,13]$ and allows CSA to be still in the game and in par with SSA and CEA. CEA on the contrary provides a segmental neural blockade and requires high volumes of local anaesthetics (LA). There always remains a concern for systemic LA absorption and toxicity. Also, when it comes to surgeries involving the hip, which require good muscle relaxation, CSA has shown to provide better block characteristics [11]. With substantial advantages, CSA has also made its way in labour analgesia practice as well [14].

CSA was employed in this case considering the ill effects GA may have on this patient with pre-existing poor pulmonary status. Also, the challenges associated with the difficult airway of this patient could be avoided with a successful neuraxial block. The anaesthetic management of this patient demanded a sound anaesthesia with minimal cardiovascular and respiratory interference. So, undertaking CSA would meet these goals by its ability to titrate the LA with the level of blockade. Various studies have reported better hemodynamic conditions with CSA in comparison to SSA for hip fracture repair $[15,16]$. The presence of an indwelling catheter would also allow for LA to be topped up in case of extended duration of surgery and postoperative pain management.

Spinal deformities in the form of accentuated lumbar lordosis, thoracic scoliosis, and narrowed epidural and intrathecal spaces may be encountered in patients with skeletal dysplasia $[3,17]$. These deformities pose a hindrance to a 
successful neuraxial block, thereby increasing the risks of complications such as repeated dural puncture, unpredictable LA spread, high block level, and difficult catheter placement $[4-7,18]$. These anatomical features along with short stature make determination of appropriate volume and dose of LA difficult. So, a titratable form of neuraxial anaesthetic techniques such as an epidural, combined spinal epidural (CSE), or continuous spinal may prove to be more suitable in such cases $[18,19]$.

Once the catheter was in place, we used a low volume of hyperbaric bupivacaine considering the uncertainty lying behind the drug doses and its spread in our patient. Crawford and Dutton used $1 \mathrm{~mL}$ of $0.5 \%$ plain bupivacaine in a patient with achondroplastic dwarfism titrating it over 30 to $45 \mathrm{~min}$ to achieve a T4 level blockade [19]. Dresner and Maclean successfully provided CSA for a caesarean section in a patient with Klippel-Feil syndrome with severe kyphoscoliosis. They used a total of $6.25 \mathrm{mg}$ heavy bupivacaine, $7.5 \mathrm{mg}$ of plain bupivacaine, and $10 \mathrm{mcg}$ of fentanyl over $20 \mathrm{~min}$, without any significant effects on cardiorespiratory function [20]. Okutomi et al. used a total of $7 \mathrm{mg}$ of hyperbaric bupivacaine in titration for a caesarean section using CSA [21]. This evidence favours towards a low volume induction technique in conjunction to intraoperative LA titration, when uncertainty prevails as in this case.

\section{Conclusions}

Continuous spinal anaesthesia is a versatile technique of neuraxial anaesthesia and an indispensable armamentarium of anaesthesiologists. Its inherent potential to provide superior neural blockade even with small doses of a local anaesthetic ensures delivery of safe, reliable, and well-controlled anaesthesia, especially in situation like ours where hemodynamic stability is crucial.

\section{Abbreviations}

$\begin{array}{ll}\text { CSA: } & \text { Continuous spinal anaesthesia } \\ \text { ED: } & \text { Emergency department } \\ \text { GCS: } & \text { Glasgow coma scale } \\ \text { Mm of Hg: } & \text { Millimeters of mercury } \\ \text { RT PCR: } & \text { Reverse transcriptase polymerase chain } \\ & \text { reaction } \\ \text { SARS-CoV- } & \text { Severe acute respiratory syndrome } \\ \text { 2: } & \text { coronavirus } 2 \\ \text { Cm: } & \text { Centimetres } \\ \text { kg: } & \text { Kilograms } \\ \text { PFT: } & \text { Pulmonary function test } \\ \text { ASA: } & \text { American Society of Anaesthesiologists } \\ \text { Inj: } & \text { Injection } \\ \text { Mg: } & \text { Milligram } \\ \text { Mcg: } & \text { Microgram } \\ \text { CSF: } & \text { Cerebrospinal fluid } \\ \text { Ml: } & \text { Milliliters } \\ \text { GA: } & \text { General anaesthesia } \\ \text { SSA: } & \text { Single-shot spinal anaesthesia } \\ \text { CEA: } & \text { Continuous epidural anaesthesia } \\ \text { LA: } & \text { Local anaesthesia } \\ & \end{array}$

CSE: Combined spinal epidural.

\section{Data Availability}

The data used to support findings of this study are included in the article.

\section{Consent}

Consent for the study was obtained.

\section{Disclosure}

This study was performed as a part of employment of the authors at the National Trauma Centre.

\section{Conflicts of Interest}

The authors declare they have no conflicts of interest.

\section{Acknowledgments}

The authors would like thank Dr Radeep Singh and Dr Surendra Maharjan for their assistance and support.

\section{References}

[1] C. A. Bacino, Skeletal Dysplasias: Approach to EvaluationUpToDate, Waltham, MA, USA, 2020.

[2] Zanelli S. A., Skeletal Dysplasia [Internet], Medscape, 2020, https://emedicine.medscape.com/article/943343-overview.

[3] K. K. White, V. Bompadre, M. J. Goldberg et al., "Best practices in peri-operative management of patients with skeletal dysplasias," American Journal of Medical Genetics Part A, vol. 173, no. 10, pp. 2584-2595, 2017.

[4] J. R. Brimacombe and J. A. Caunt, "Anaesthesia in a gravid achondroplastic dwarf," Anaesthesia, vol. 45, no. 2, pp. 132-134, 1990.

[5] G. J. Wardall and W. T. Frame, "Extradural anaesthesia for caesarean section in achondroplasia," British Journal of Anaesthesia, vol. 64, no. 3, pp. 367-370, 1990.

[6] S. E. Cohen, "Anesthesia for cesarean section in achondroplastic dwarfs," Anesthesiology, vol. 52, no. 3, pp. 264-266, 1980.

[7] M. J. Morrow and I. H. Black, "Epidural anaesthesia for caesarean section in an achondroplastic dwarf," British Journal of Anaesthesia, vol. 81, no. 4, pp. 619-621, 1998.

[8] J. S. DeRenzo, M. C. Vallejo, and S. Ramanathan, "Failed regional anesthesia with reduced spinal bupivacaine dosage in a parturient with achondroplasia presenting for urgent cesarean section," International Journal of Obstetric Anesthesia, vol. 14, no. 2, pp. 175-178, 2005.

[9] J. Guay, M. J. Parker, P. R. Gajendragadkar, and S. Kopp, "Anaesthesia for hip fracture surgery in adults," The Cochrane Database of Systematic Reviews, vol. 2, no. 2, p. CD000521, 2016.

[10] S. G. Memtsoudis, C. Cozowicz, J. Bekeris et al., "Anaesthetic care of patients undergoing primary hip and knee arthroplasty: consensus recommendations from the International Consensus on Anaesthesia-Related Outcomes after Surgery group (ICAROS) based on a systematic review and metaanalysis," British Journal of Anaesthesia, vol. 123, no. 3, pp. 269-287, 2019. 
[11] J. M. Moore, "Continuous spinal anesthesia," American Journal of Therapeutics, vol. 16, no. 4, pp. 289-294, 2009.

[12] E. A. Lux, "Continuous spinal anesthesia for lower limb surgery: a retrospective analysis of 1212 cases," Local and Regional Anesthesia, vol. 5, pp. 63-67, 2012.

[13] I. Veličković, B. Pujic, C. W. Baysinger, and C. L. Baysinger, "Continuous spinal anesthesia for obstetric anesthesia and analgesia," Frontiers in Medicine, vol. 4, p. 133, 2017.

[14] J.-W. Ji, M.-J. Xu, B. Han, L. Chen, and X.-J. Mu, "Feasibility study on continuous spinal analgesia in all stages of labor," Chinese Medical Journal, vol. 133, no. 5, pp. 618-620, 2020.

[15] J. F. Favarel-Garrigues, F. Sztark, M. E. Petitjean, M. Thicoïpé, P. Lassié, and P. Dabadie, "Hemodynamic effects of spinal anesthesia in the elderly: single dose versus titration through a catheter," Anesthesia \& Analgesia, vol. 82, no. 2, pp. 312-316, 1996.

[16] V. Minville, O. Fourcade, D. Grousset et al., "Spinal anesthesia using single injection small-dose bupivacaine versus continuous catheter injection techniques for surgical repair of hip fracture in elderly patients," Anesthesia \& Analgesia, vol. 102, no. 5, pp. 1559-1563, 2006.

[17] N. Nisa, P. Khanna, and D. Jain, "Anaesthetic management of an achondroplastic dwarf with difficult airway and spine for total hip replacement: a case report," General Medicine, vol. 4, no. 2, p. 227, 2016

[18] R. D. A. McArthur, "Obstetric anaesthesia in an achondroplasic dwarf at a regional hospital," Anaesthesia and Intensive Care, vol. 20, no. 3, pp. 376-378, 1992.

[19] M. Crawford and D. A. Dutton, "Spinal anaesthesia for caesarean section in an achondroplastic dwarf," Anaesthesia, vol. 47, no. 11, p. 1007, 1992.

[20] M. R. Dresner and A. R. Maclean, "Anaesthesia for Caesarean section in a patient with Klippel-Feil syndrome," Anaesthesia, vol. 50, no. 9, pp. 807-809, 1995.

[21] T. Okutomi, M. Saito, M. Koura, and S. Hoka, "Spinal anesthesia using a continuous spinal catheter for cesarean section in a parturient with prior surgical correction of scoliosis," Journal of Anesthesia, vol. 20, no. 3, pp. 223-226, 2006. 